\title{
CdSe:Te Nanocrystals: Band-Edge versus Te-Related Emission
}

Thomas Franzl, Josef Müller, Thomas A. Klar, Andrey L. Rogach,* and Jochen Feldmann

Photonics and Optoelectronics Group, Physics Department \& Center for Nanoscience (CeNS), Ludwig-Maximilians-Universität München, 80799 Munich, Germany

Dmitri V. Talapin** and Horst Weller

Institute of Physical Chemistry, University of Hamburg, Grindelallee 117, 20146 Hamburg, Germany

${ }^{\#}$ Present Address: The Molecular Foundry, Lawrence Berkeley National Laboratory, Berkeley, CA, USA 


\section{Supporting Information}
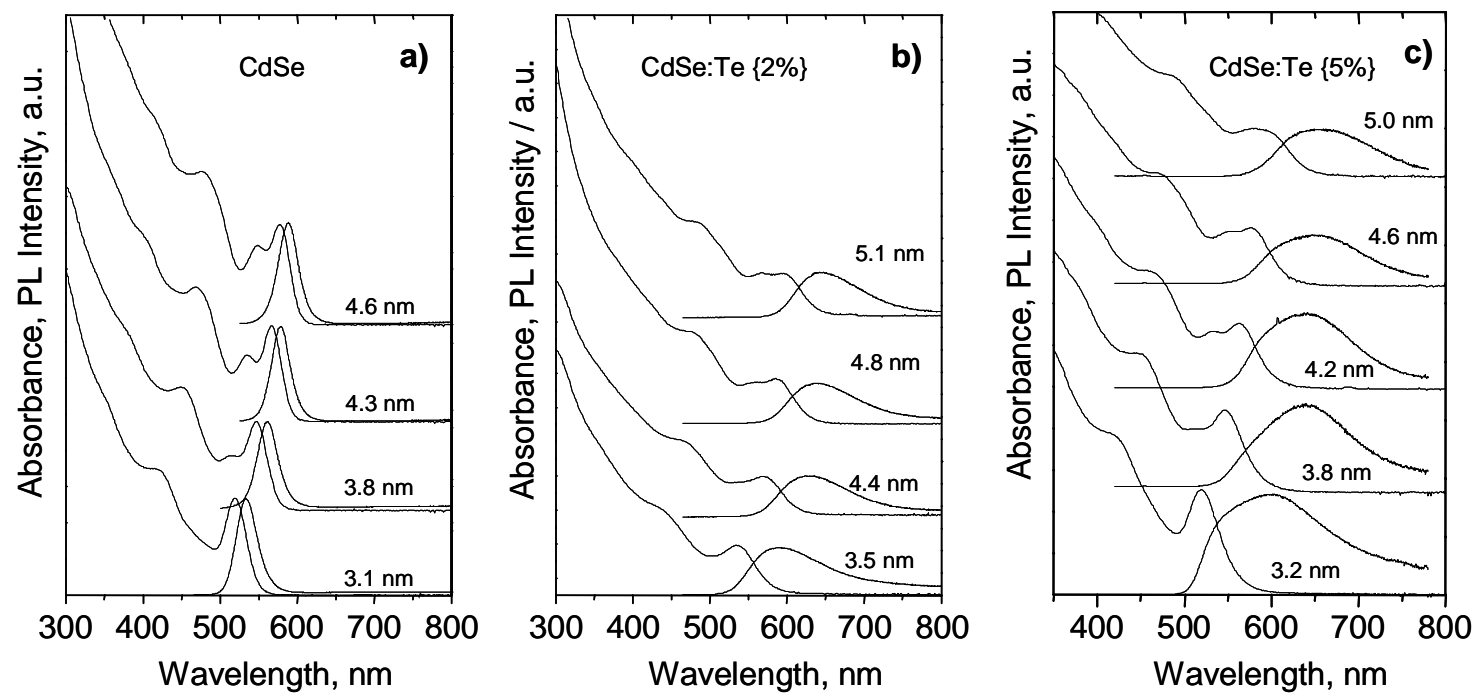

Figure S1. Growth dynamics of CdSe (a), CdSe:Te \{2\%\} (b), and CdSe:Te \{5\%\}(c) NCs monitored by absorption and PL spectra.

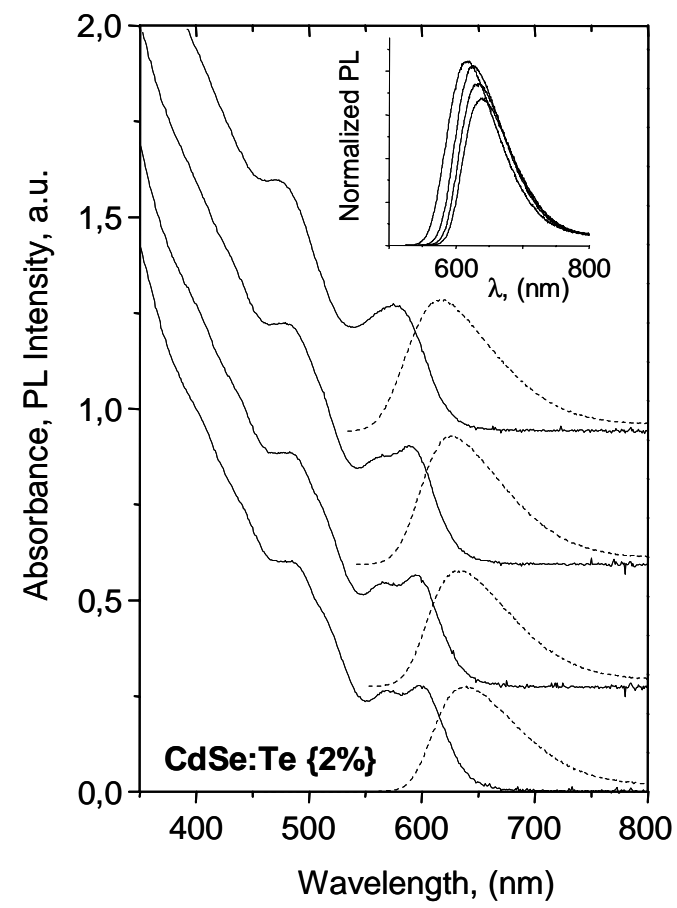

Figure S2. Absorption and PL spectra of size-selected fractions of $4.8 \mathrm{~nm}$ CdSe:Te\{2\%\} NCs.

The inset shows PL spectra of size-selected fractions with the PL intensity normalized to absorbance at the excitation wavelength $(400 \mathrm{~nm})$. 


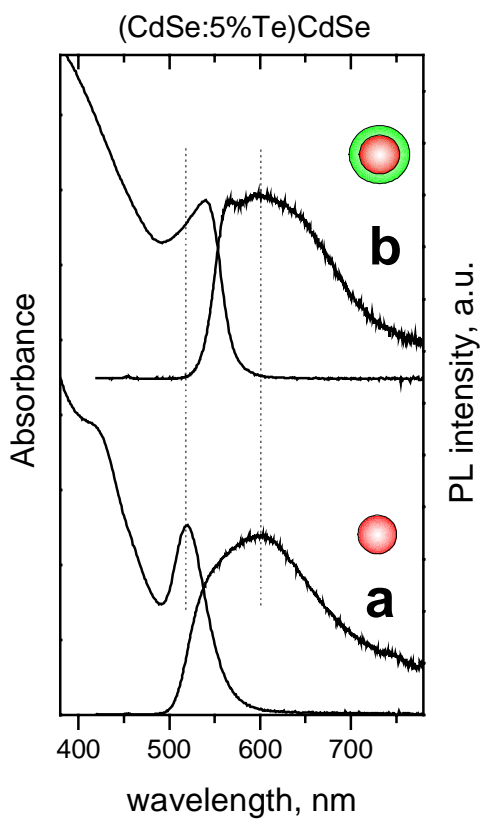

Figure S3. Absorption and PL spectra of CdSe:Te nanocrystals (a) before and (b) after growing the shell of pure CdSe.

Our analysis is based on the hypothesis that Te atoms incorporated into CdSe lattice during nanocrystal growth. However, the other possibility could be that Te adsorbs at the nanocrystal surface and affects the luminescent properties without being integrated into the crystal lattice. To test this scenario, we thoroughly washed as-synthesized CdSe:Te nanocrystals from any residual Te present in the solution by precipitating the nanocrystals with methanol and redissolving them in pure toluene several times. Next, we dissolved the nanocrystals in HDATOPO-TOP mixture and grew the layer of CdSe around Te-doped CdSe cores by adding dimethylcadmium and TOPSe to the solution at $240^{\circ} \mathrm{C}$. The shell of pure CdSe encapsulated all Te atoms inside the nanocrystals. Figure S3 shows absorption and PL spectra of Te-doped CdSe nanocrystals before and after growing the shell of pure CdSe. Comparison of the absorption and PL spectra revealed that the large Stokes shift and broadening of the luminescence band observed in CdSe:Te nanocrystal samples are due to the incorporation of Te atoms into CdSe lattice and cannot be caused by adsorption of Te at the nanoparticle surface. 